Urologe $2021 \cdot 60: 222-225$

https://doi.org/10.1007/s00120-020-01391-7

Online publiziert: 17 . November 2020

(c) Der/die Autor(en) 2020

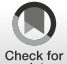

\author{
Maximilian Haider ${ }^{1,2}$ - Evi Comploj ${ }^{3,4} \cdot$ Aybike Hofmann' ${ }^{1}$ Wolfgang H. Rösch ${ }^{1}$ \\ ${ }^{1}$ Klinik für Kinderurologie in Kooperation mit der Universität Regensburg, Krankenhaus Barmherzige \\ Brüder, Klinik St. Hedwig, Regensburg, Deutschland \\ ${ }^{2}$ Klinik für Urologie, Caritas Krankenhaus St. Josef, Lehrstuhl für Urologie der Universität Regensburg, \\ Regensburg, Deutschland \\ ${ }^{3}$ Abteilung für Urologie, Zentralkrankenhaus Bozen, Bozen, Italien \\ ${ }^{4}$ Landesfachhochschule für Gesundheitsberufe, Claudiana-Research, Bozen, Italien
}

\title{
Der juvenile Granulosazelltumor - der Hodentumor der Kleinsten
}

\section{Aktuelle Handlungsempfehlungen anhand von drei Kasuistiken}

daraufhin belassen und intraskrotal pexiert. Im weiteren Verlauf Beendigung der Antibiose bei unauffälligem Trachealsekret. Verlegung des Patienten am 4. postoperativen Tag auf Normalstation und Entlassung am 5. postoperativen Tag.

Die endgültige Histologie ergab aus dem intraoperativen Präparat einen Granulosazelltumor vom juvenilen Typ (JGZT). Die daraufhin veranlasste Chromosomenanalyse erbringt einen unauffälligen männlichen Chromosomensatz.

Im 4. Lebensmonat wird bei klinisch und laborchemisch (einschließlich Tumormarker) unauffälligen Befunden, aber sonographisch unregelmäßiger Parenchymtextur des verbliebenen Resthodengewebes eine skrotale Orchiektomie links durchgeführt. Histologisch bestätigt sich erneut der nun in toto resezierte juvenile Granulosazelltumor. Gleichzeitig findet eine Fertilitätsbeurteilung am Semidünnschnitt statt. Hier zeigen sich vorwiegend fetale A-pale-Spermatogonien und nur sehr spärlich vom A-darkTyp (reife Formen).

Der Patient ist nun seit knapp 11 Jahren in Nachsorge ohne Hinweis für ein Rezidiv oder Metastasen.

\section{Fall B}

Bei einem männlichen Neugeborenen im EU-Ausland wird unmittelbar postnatal eine Hydrocele testis diagnostiziert. Im
Alter von 10 Wochen wird bei verändertem Tastbefund eine Sonographie durchgeführt, hier ergibt sich der Verdacht auf einen malignen Hodentumor. Im MRTBecken mit Kontrastmittel erhärtet sich der Befund. Ein Hinweis auf eine Filialisierung besteht nicht. Mit 12 Wochen wird der Patient erstmals urologisch vorstellig. Bei der körperlichen Untersuchung findet sich eine schmerzlose, tastsuspekte Raumforderung des rechten Hodens. In der Sonographie ist das Parenchym des rechten Hodens nahe$\mathrm{zu}$ vollständig durchsetzt von einer inhomogenen, gut durchbluteten, malignitätssuspekten und von den peritestikulären Strukturen abgrenzbaren Raumforderung. Die Tumormarker ergeben ein erhöhtes AFP mit 121,4ng/ml (Norm im Alter $>10$ Monate: $3-28 \mathrm{ng} / \mathrm{ml}$ ) bei normwertigem $\beta$-HCG und LDH. Es erfolgt daraufhin die inguinale Freilegung. Intraoperativ kann der Tumor nur sonographisch vom spärlichen Hodenrest differenziert werden, deshalb Entschluss zur Orchiektomie. Der Patient wird am ersten postoperativen Tag beschwerdefrei entlassen.

Histologisch wird in der konventionellen Mikroskopie ein multizystischer juveniler Granulosazelltumor gesehen, dieser wird durch die Immunhistochemie und die spätere Referenzpathologie bestätigt. 

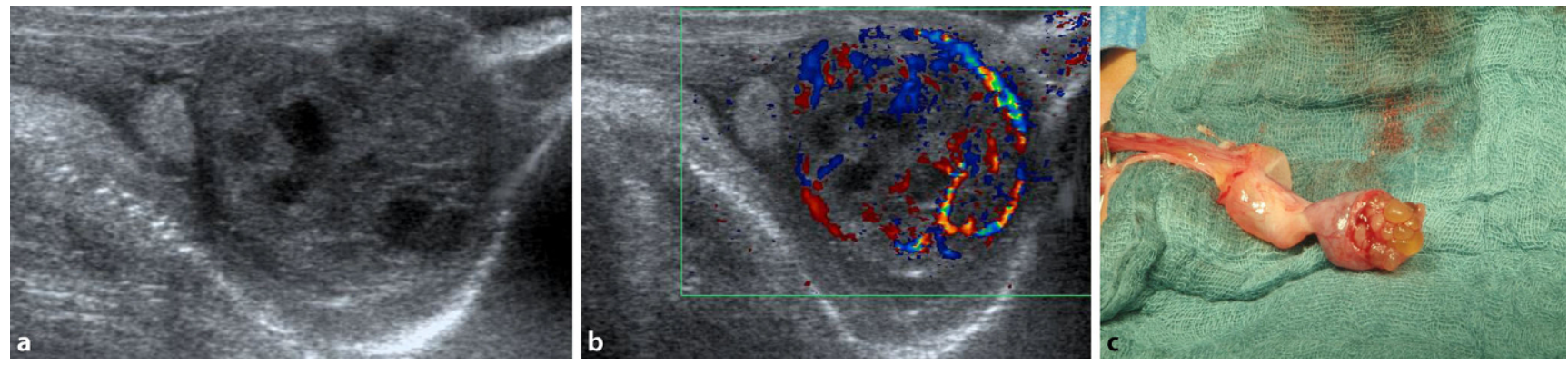

Abb. $1 \Delta$ Fall A: a Hodensonogramm (B-Bild), b Hodensonogramm (Farbduplex), c intraoperativer Befund
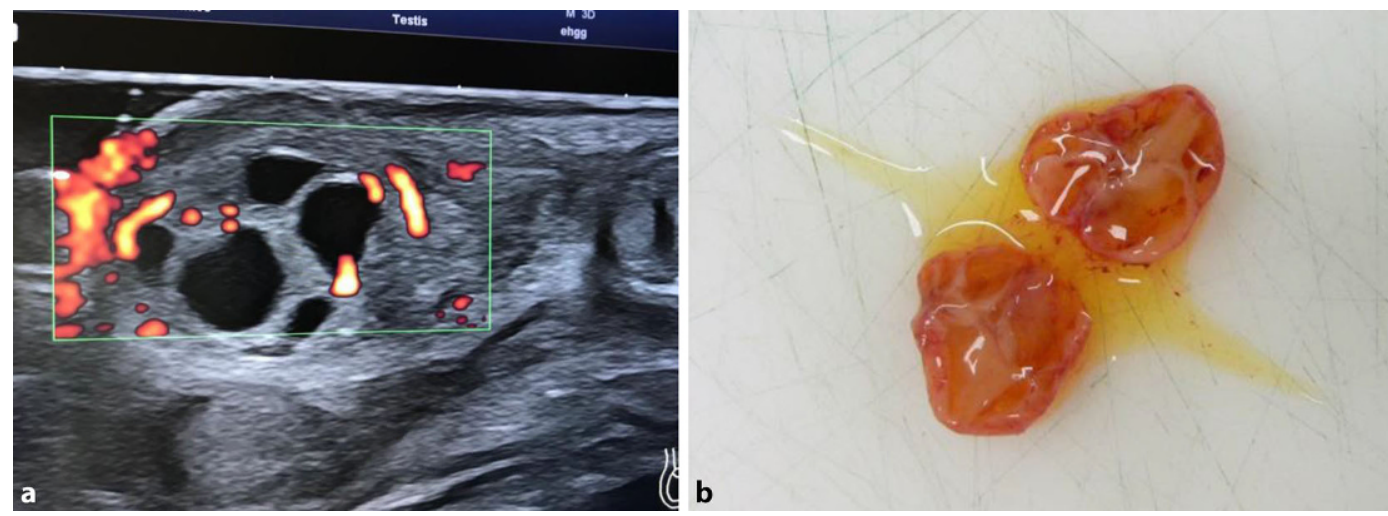

Abb. $2 \triangleleft$ Fall C: a Hodensonogramm (Power-Doppler), b makropathologischer Befund (b mit freundl. Genehmigung durch Dr. Esther Hanspeter und Dr. Domenico Damiani, Bozen, all rights reserved)

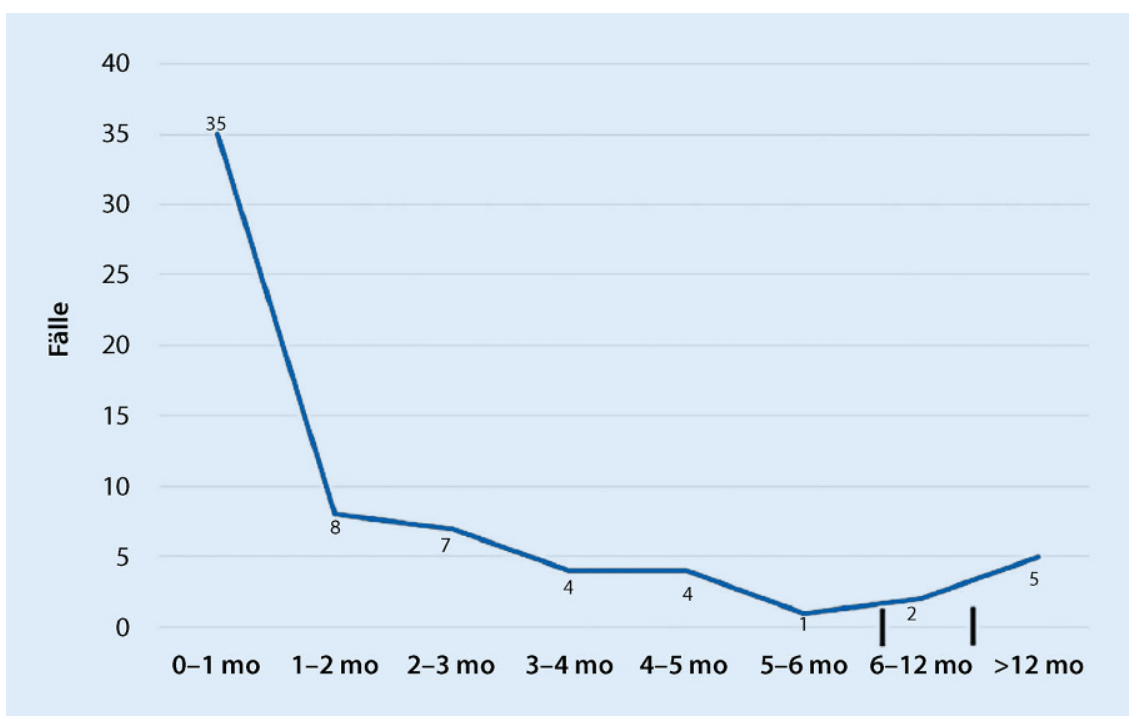

Abb. $3 \Delta$ Altersverteilung von 66 JGZT-Patienten (juveniler Granulosazelltumor, mo Monate). (Aus [15])

\section{Fall C}

Ein Junge wird reif $(39+2$ SSW $)$ mit Geburtsgewicht von $3530 \mathrm{~g}$ geboren. Im Rahmen der U2 fällt ein vergrößertes linkes Hemiskrotum mit palpatorisch verhärtetem Hoden auf. In der Sonographie ( Abb. 2a) zeigen sich multiple zystische Veränderungen des Hodenpa- bestätigt. Entlassung am Folgetag. Vier Monate postoperativ zeigt sich ein klinisch und sonographisch unauffälliger Befund.

\section{Diskussion}

Der JGZT ist ein Tumor des Neugeborenen und des frühen Säuglingsalters. Damit nimmt er unter den kindlichen $\mathrm{Tu}$ morentitäten inklusive des Dottersacktumors eine Sonderrolle ein ( Abb. 3; [15]). Gelegentlich kann die Diagnose bereits intrauterin gestellt werden [16].

Diagnostisch steht die Sonographie im Vordergrund. Bei passender Vorgeschichte und klinischem Bild ist durch den sonographischen Nachweis einer multizystischen, klar abgrenzbaren, intratestikulären Raumforderung das Vorliegen eines JGZT sehr wahrscheinlich. Gleichwohl ist die Diagnosestellung keineswegs trivial. Im Fall A wurde das Bild einer älteren Hodentorsion vorgetäuscht mit echoarmen, wabenartigen Strukturen, die durchaus auch hämorrhagischen Einblutungen oder bereits nekrotischen Arealen bei stattgehabter Torsion hätten entsprechen können. Im Fall B wurde der Sonographiebefund einem 
malignen Prozess zugeordnet. Differentialdiagnostisch muss neben anderen Tumorentitäten (z.B. Dottersacktumor) eine zystische Dysplasie des Rete testis erwogen werden [6]. Da sowohl Seminome als auch Chorionkarzinome vor der Pubertät praktisch nie auftreten, hat $\beta$-HCG als Tumormarker im Kindesalter keine Bedeutung. Die Interpretation des Hodentumormarkers AFP ist aufgrund der physiologisch hohen Werte im frühen Säuglingsalter oft schwierig. Die zur Verfügung stehenden altersabhängigen Normwertbereiche für die ersten 6 Monate sind groß. Eine zuverlässigere Aussage zur präoperativen Differenzierung zum Dottersacktumor ist erst ab dem 6. Lebensmonat möglich $[11,13]$. Die Rolle neuer Tumormarker (miRNA) scheint auch im pädiatrischen Bereich vielversprechend [9], ist bislang jedoch noch nicht für die Routine etabliert. Ein klassisches Tumorstaging wie im Erwachsenenalter mit Röntgen, CT und MRT ist im Kindesalter nicht indiziert und bleibt nur den Fällen mit histologisch nachgewiesenem Dottersacktumor vorbehalten.

Therapie der Wahl ist heute die zeitnahe inguinale Freilegung des Hodens mit chirurgischer Resektion der Raumforderung. Grundsätzlich ist ein Organerhalt anzustreben [5]. Jedoch werden auch in der Literatur Fälle beschrieben, wo aufgrund eines nur spärlich oder gar nicht mehr vorhandenen Restparenchyms eine Orchiektomie unumgänglich war (z. B. [12]), vergleichbar mit unserem Fall B. Stets sollte aber die Indikation zur Orchiektomie im Säuglings- und Kindesalter sehr streng gestellt werden, nicht zuletzt da im Rahmen anderer benigner kindlicher Tumorentitäten gezeigt werden konnte, dass nach organerhaltender Resektion auch bei ausgeprägten Befunden ein enormes Aufholpotential des betroffenen Hodens bis hin zur Restitution einer symmetrischen Hodengröße gegeben ist [12].

Die Schnellschnittdiagnostik gilt heute auch im Kindesalter als obligat [13]. Allerdings kann in der konventionellen Histologie die Differenzierung des JGZT zu einem Dottersacktumor gelegentlich schwierig sein. Zur endgültigen Diagnosestellung sollte deshalb stets zusätzlich eine immunhistochemische Aufarbeitung erfolgen [4]. Gerade vor diesem Hintergrund ist es aber entscheidend, dass dem Operateur der JGZT als Differentialdiagnose präsent ist, um eine schnellschnittbedingte Orchiektomie im Sinne einer Übertherapie zu vermeiden.

Da mit hoher Wahrscheinlichkeit von einer benignen Läsion auszugehen ist, wird gelegentlich auch ein primär transskrotaler Zugang diskutiert [1]. Der inguinale Zugang ist jedoch nicht wesentlich invasiver, zusätzlich kann aber ein in diesem Alter oft begleitender noch offener Processus vaginalis mitversorgt werden; im seltenen Falle einer unerwartet malignen Raumforderung kann eine hohe Absetzung des Samenstrangs erfolgen und es werden zudem keine weiteren Metastasierungswege eröffnet.

Der JGZT des Hodens ist eine gutartige Raumforderung und verhält sich anders als der adulte Granulosazelltumor oder der JGZT des Ovars. Rezidive oder Filialisierungen sind nicht beschrieben. Die Patienten zeigen i.d. R. keine Auffälligkeiten, die auf eine hormonelle Aktivität des Tumors schließen lassen würden [7]. In der Literatur sind geschlechtschromosomale Auffälligkeiten bei Patienten mit JGZT des Hodens ausschließlich bei konkomitanten weiteren klinischen Auffälligkeiten des äußeren Genitales (ausgeprägte/penoskrotale Hypospadie, „ambiguous genitalia“ oder Hinweise auf ein komplexeres Fehlbildungssyndrom) beschrieben. Hier zeigt sich jedoch ein verhältnismäßig häufiges Vorkommen [2, 3, 8, 10, 14, 17]. Anders als in manchen Lehrbüchern etwas unscharf formuliert, ist daher eine Chromosomenanalyse allein durch die histologische Diagnose JGZT bei ansonsten gesunden Patienten heute nicht mehr indiziert.

Die jährliche Nachsorge beschränkt sich auf die klinische und sonographische Kontrolle, eine weitere Bildgebung, Tumormarker oder Hormondiagnostik sind standardmäßig nicht notwendig.

Die systematische und konsequente Erfassung der kindlichen testikulären Raumforderungen jeder Dignität in Korrelation zum Sonographiebefund in einem Tumorregister wäre wünschenswert. Dies würde zu einer belastbareren
Urologe $2021 \cdot 60: 222-225$

https://doi.org/10.1007/s00120-020-01391-7

(c) Der/die Autor(en) 2020

M. Haider - E. Comploj · A. Hofmann -

W. H. Rösch

Der juvenile

Granulosazelltumor - der

Hodentumor der Kleinsten.

Aktuelle

Handlungsempfehlungen anhand von drei Kasuistiken

Zusammenfassung

Der juvenile Granulosazelltumor (JGZT) des Hodens ist eine relevante Differentialdiagnose testikulärer Raumforderungen des Säuglingsalters. Es handelt sich um eine benigne Läsion, die Therapie ist rein chirurgisch. Metastasierungen oder Rezidive sind nicht bekannt. Am Beispiel von drei Kasuistiken wird das aktuelle diagnostische und therapeutische Management vorgestellt und diskutiert.

Schlüsselwörter

Hodentumor - Keimzell-Stroma-Tumoren . Hodensonographie · Pädiatrische Tumoren . Kinderurologie

\section{Juvenile granulosa cell tumor-Testicular tumor of the very young. Report of three cases and contemporary recommendations}

\section{Abstract}

Juvenile granulosa cell tumor of the testis is a relevant differential diagnosis regarding testicular neoplasia of the very young. This benign lesion requires surgical treatment. Metastases or recurrences have not been described in literature. We present three different cases and give recommendations for diagnosis and treatment.

Keywords

Testicular cancer - Testicular sex cord stroma tumor - Scrotal ultrasound - Pediatric tumor . Pediatric urology

Risikostratifizierung für diese Patienten führen. Neue Tumormarker (miR371 373 und miR-302) werden wahrscheinlich auch im Kindesalter die Differenzierung von benignen und malignen Prozessen und die Entscheidung zum Organerhalt erleichtern [9]. 


\section{Korrespondenzadresse}

Dr. med. Maximilian Haider
Klinik für Kinderurologie
in Kooperation mit der
Universität Regensburg,
Krankenhaus Barmherzige
Brüder, Klinik St. Hedwig
Steinmetzstraße 1-3,
93049 Regensburg,
Deutschland
maximilian.haider@ukr.de

Funding. Open Access funding enabled and organized by Projekt DEAL.

\section{Einhaltung ethischer Richtlinien}

Interessenkonflikt. M. Haider, E. Comploj, A. Hofmann und W.H. Rösch geben an, dass kein Interessenkonflikt besteht.

Für diesen Beitrag wurden von den Autoren keine Studien an Menschen oder Tieren durchgeführt. Für die aufgeführten Studien gelten die jeweils dort angegebenen ethischen Richtlinien. Für Bildmaterial oder anderweitige Angaben innerhalb des Manuskripts, über die Patienten zu identifizieren sind, liegt von ihnen und/oder ihren gesetzlichen Vertretern eine schriftliche Einwilligung vor.

Open Access. Dieser Artikel wird unter der Creative Commons Namensnennung 4.0 International Lizenz veröffentlicht, welche die Nutzung, Vervielfältigung, Bearbeitung, Verbreitung und Wiedergabe in jeglichem Medium und Format erlaubt, sofern Sie den/die ursprünglichen Autor(en) und die Quelle ordnungsgemäß nennen, einen Link zur Creative Commons Lizenz beifügen und angeben, ob Änderungen vorgenommen wurden.

Die in diesem Artikel enthaltenen Bilder und sonstiges Drittmaterial unterliegen ebenfalls der genannten Creative Commons Lizenz, sofern sich aus der Abbildungslegende nichts anderes ergibt. Sofern das betreffende Material nicht unter der genannten Creative Commons Lizenz steht und die betreffende Handlung nicht nach gesetzlichen Vorschriften erlaubt ist, ist für die oben aufgeführten Weiterverwendungen des $\mathrm{Ma}$ terials die Einwilligung des jeweiligen Rechteinhabers einzuholen.

Weitere Details zur Lizenz entnehmen Sie bitte der Lizenzinformation auf http://creativecommons.org/ licenses/by/4.0/deed.de.

\section{Literatur}

1. Bulotta AL, Molinaro F, Angotti R et al (2012) Juvenile granulosa cell tumor of the testis. Prenatal diagnosis and prescrotal approach. Ital J Pediatr 38:67

2. Chan JK, Chan VS, Mak KL (1990) Congenital juvenile granulosa cell tumour of the testis. Report of a case showing extensive degenerative changes. Histopathology 17(1):75-80
3. Claros OR, Sakai AT, Consolmagno H et al (2014) Granulosa cell tumor of the testis in a newborn. Autops Case Rep 4(1):39-44

4. Cornejo KM, Frazier L, Lee RS et al (2015) Yolk Sac tumor of the testis in infants and children. A clinicopathologic analysis of 33 cases. Am J Surg Pathol 39(8):1121-1131

5. Cosentino M, Algaba F, Saldana L et al (2014) Juvenile granulosa cell tumor of the testis. A bilateral and synchronous case. Should testis-sparing surgery be mandatory? Urology 84(3):694-696

6. Gelas T, Margain Deslandes L, Mestrallet G et al (2016) Spontaneous regression of suspected cystic dysplasia of the rete testis in three neonates. J Pediatr Urol 12(6):387.e1-387.e4

7. Kao C-S, Cornejo KM, Ulbright TM et al (2015) Juvenile granulosa cell tumors of the testis. A clinicopathologic study of 70 cases with emphasis on its wide morphologic spectrum. Am J Surg Pathol 39(9):1159-1169

8. Kos M, Nogales FF, Kos M et al (2005) Congenital juvenile granulosa cell tumor of the testis in a fetus showing full 69,XXY triploidy. Int J Surg Pathol 13(2):219-221

9. Murray MJ, Huddart RA, Coleman N (2016) The present and future of serum diagnostic tests for testicular germ cell tumours. Nat Rev Urol 13(12):715-725

10. Raju U, Fine G, Warrier R et al (1986) Congenital testicular juvenile granulosa cell tumor in a neonate with X/XY mosaicism. Am J Surg Patho 10(8):577-583

11. Ross JH, Rybicki L, Kay R (2002) Clinical behavior and a contemporary management algorithm for prepubertal testis tumors. A summary of the prepubertal testis tumor registry. J Urol 168(4):1675-1678 (discussion 1678-9)

12. Shukla AR, HuffDS, Canning DA etal (2004) Juvenile granulosa cell tumor of the testis: contemporary clinical management and pathological diagnosis. JUrol 171(5):1900-1902

13. Stein R, Dürken M, Zahn K et al (2020) Hodentumoren bei präpubertären Jungen - Organerhalt häufiger möglich als gedacht. Urologe 59(3):278-283

14. Tanaka Y, Sasaki Y, Tachibana K et al (1994) Testicular juvenile granulosa cell tumor in an infant with $X / X Y$ mosaicism clinically diagnosed as true hermaphroditism. Am J Surg Pathol 18(3):316-322

15. Ulbright TM, Young RH (2014) Testicular and paratesticular tumors and tumor-like lesions in the first 2 decades. Semin Diagn Pathol 31 (5):323-381

16. Vatta F, Raffaele A, Pasqua N et al (2019) Juvenile granulosa cell tumor of the testis. Prenatal diagnosis and management. European J Pediatr Surg Rep 7(1):e93-e95

17. Young RH, Lawrence WD, Scully RE (1985) Juvenile granulosa cell tumor-another neoplasm associated with abnormal chromosomes and ambiguous genitalia. A report of three cases. Am J Surg Pathol 9(10):737-743
Hier steht eine Anzeige. Springer 